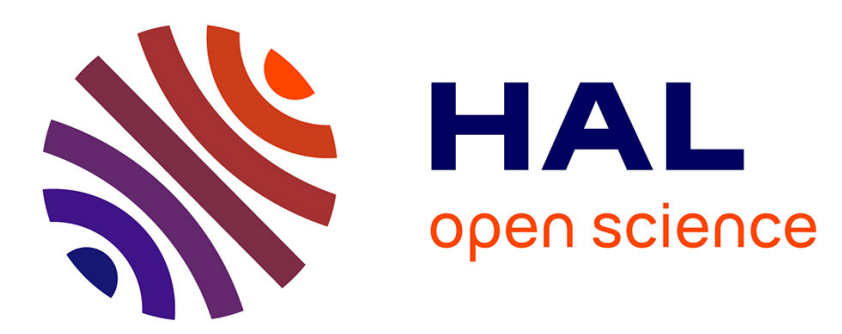

\title{
Hypernode Graphs for Spectral Learning on Binary Relations over Sets
}

\author{
Thomas Ricatte, Rémi Gilleron, Marc Tommasi
}

\section{To cite this version:}

Thomas Ricatte, Rémi Gilleron, Marc Tommasi. Hypernode Graphs for Spectral Learning on Binary Relations over Sets. Conférence Francophone sur l'Apprentissage Automatique (Cap 2014), Jul 2014, Saint-Etienne, France. 10.1007/978-3-662-44851-9_42 . hal-01104618

\section{HAL Id: hal-01104618 \\ https://inria.hal.science/hal-01104618}

Submitted on 18 Jan 2015

HAL is a multi-disciplinary open access archive for the deposit and dissemination of scientific research documents, whether they are published or not. The documents may come from teaching and research institutions in France or abroad, or from public or private research centers.
L'archive ouverte pluridisciplinaire HAL, est destinée au dépôt et à la diffusion de documents scientifiques de niveau recherche, publiés ou non, émanant des établissements d'enseignement et de recherche français ou étrangers, des laboratoires publics ou privés. 


\title{
Hypernode Graphs for Spectral Learning on Binary Relations over Sets*
}

\author{
Thomas Ricatte ${ }^{1}$, Rémi Gilleron ${ }^{2}$, and Marc Tommasi ${ }^{2}$ \\ ${ }^{1}$ SAP Research, Paris \\ ${ }^{2}$ Lille University, LIFL and Inria Lille
}

18 janvier 2015

\section{Résumé}

We introduce hypernode graphs as (weighted) binary relations between sets of nodes : a hypernode is a set of nodes, a hyperedge is a pair of hypernodes, and each node in a hypernode of a hyperedge is given a non negative weight that represents the node contribution to the relation. Hypernode graphs model binary relations between sets of individuals while allowing to reason at the level of individuals. We present a spectral theory for hypernode graphs that allows us to introduce an unnormalized Laplacian and a smoothness semi-norm. In this framework, we are able to extend existing spectral graph learning algorithms to the case of hypernode graphs. We show that hypernode graphs are a proper extension of graphs from the expressive power point of view and from the spectral analysis point of view. Therefore hypernode graphs allow to model higher order relations while it has been shown in [1] that it is not the case for (classical) hypergraphs. In order to prove the capabilities of the model, we represent multiple players games with hypernode graphs and introduce a novel method to infer skill ratings from the game outcomes. We show that spectral learning algorithms over hypernode graphs obtain competitive results with skill ratings specialized algorithms such as Elo duelling and TrueSkill.

Mots-clef : Graphs, Hypergraphs, Semi Supervised Learning, Multiple Players Games.

* This work was supported by the French National Research Agency (ANR). Project Lampada ANR-09-EMER-007.

\section{Introduction}

Graphs are commonly used as a powerful abstract model to represent binary relationships between individuals. Binary relationships between individuals are modeled by edges between nodes. This is for instance the case for social networks with the friendship relation, or for computer networks with the connection relation. The hypergraph formalism (see [2]) has been introduced for modeling problems where relationships are no longer binary, that is when they involve more than two individuals. Hypergraphs have been used for instance in bioinformatics ([10]), computer vision ([15]) or natural language processing [3]. But, graphs and hypergraphs are limited when one has to consider relationships between sets of individual objects. A typical example is the case of multiple players games where a game can be viewed as a relationship between two teams of multiple players. Other examples include relationships between groups in social networks or between clusters in computer networks. For these problems, considering both the group level and the individual level is a requisite. For instance for multiple players games, one is interested in predicting game outcomes for games between teams as well as in predicting player skills. Graphs fail to model relationships between sets of individual objects because dependencies among sets would be lost. Hypergraphs fail to model relationships between sets of individual objects because a hyperedge does not model a relationship between sets of objects.

A first contribution of this paper is to introduce a new class of undirected hypergraphs called hypernode graphs for modeling binary relationships between sets of individual objects. A relationship between two sets of individual objects is represented by a hyperedge which is defined to be a pair of disjoint hypernodes, 


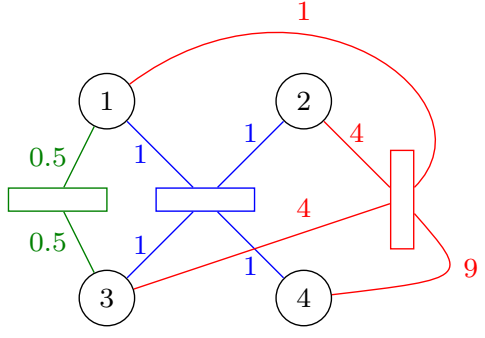

Figure 1 - A hypernode graph modeling 3 tennis games with 4 players. Each of the three hyperedges has one color and models a game for which players connected to the same long edge of a rectangle are in the same team.

where a hypernode is a set of nodes. Nodes in a hypernode of a hyperedge are given a non negative weight that represents the node contribution to the binary relationship. An example of hypernode graph is presented in Figure 1. There are four nodes that represent four tennis players and three hyperedges representing three games between teams : $\{1\}$ against $\{3\},\{1,2\}$ against $\{3,4\}$, and $\{1,4\}$ against $\{2,3\}$. For each hyperedge, each player has been given a weight which can be seen as the player's contribution. It can be noted that the hyperedge between singleton sets $\{1\}$ and $\{3\}$ can be viewed as an edge between nodes 1 and 3 with edge weight 1 . Undirected graphs are shown to be hypernode graphs where hypernodes are singleton sets.

Given a hypernode graph modeling binary relationships between sets of individuals, an important task, as said above, is to evaluate individuals by means of node labelling or node scoring functions. The second contribution of this paper is to propose machine learning algorithms in the semi-supervised, batch setting on hypernode graphs for predicting node labels or node scores. To this aim, we develop a spectral learning theory for hypernode graphs. Similarly to the case of graph spectral learning, our approach relies on the assortative mixing (or homophilic) assumption which says that two linked nodes should have the same label or similar scores. For graphs, this assumption is reflected in the choice of smooth node functions for which linked nodes get values that are close enough. For hypernode graphs, we assume an additive model, and we will say that a real-valued node function over a hypernode graph is smooth if, for linked hypernodes, the weighted sum of function values over the two node sets are close enough. As an example, let us consider the blue hyperedge in Figure 1 between the two sets
$\{1,2\}$ and $\{3,4\}$ and a real-valued node function $f$, the function $f$ is said to be smooth over the hyperedge if $f(1)+f(2)$ is close to $f(3)+f(4)$.

For defining the smoothness, we introduce an unnormalized gradient for hypernode graphs. Then, we define an unnormalized Laplacian $\Delta$ for hypernode graphs by $\Delta=G^{T} G$ where $G$ is the gradient. We show that the class of hypernode graph Laplacians is the class of symmetric positive semidefinite real-valued matrices $M$ such that $\mathbf{1} \in \operatorname{Null}(M)$, where $\operatorname{Null}(M)$ denotes the null space of $M$. This result allows to show that there exist hypernode graphs whose Laplacian matches that of a graph and that there exist hypernode graph Laplacians which are not the graph Laplacian of any graph. While it has been proved in 11 that hypergraph Laplacians can be defined from graph Laplacians using adequate graph construction. Then, the smoothness of a real-valued node function $f$ on a hypernode graph can be characterized by the smoothness semi-norm defined by $\Omega(f)=f^{T} \Delta f$. We define the kernel of a hypernode graph to be the Moore-Penrose pseudoinverse of its Laplacian. The spectral theory for hypernode graphs and its properties allow us to use spectral graph learning algorithms [14, 16, 18 for hypernode graphs.

We apply hypernode graph spectral learning to the rating of individual skills of players and to the prediction of game outcomes in multiple players games. We consider competitive games between two teams where each team is composed of an arbitrary number of players. Each game is modeled by a hyperedge and a set of games is represented by a hypernode graph. We define a skill rating function of players as a realvalued node function over the hypernode graph. And we show that finding the optimal skill rating function reduces to finding the real-valued function $s^{*}$ minimizing $\Omega(s)=s^{T} \Delta s$, where $\Delta$ is the unnormalized Laplacian of the hypernode graph. The optimal individual skill rating function allows to compute the rating of teams and to predict game outcomes for new games. We apply this learning method on real datasets of multiple player games to predict game outcomes in a semi-supervised, batch setting. Experimental results show that we obtain very competitive results compared to specialized algorithms such as Elo duelling and TrueSkill.

Related Work. Hypernode graphs that we introduced can be viewed as an undirected version of directed hypergraphs popularized by [5] where a directed hyperedge consists in an oriented relation between two sets of nodes. As far as we know, this class of directed hypergraphs has not been studied from the ma- 
chine learning point of view and no attempt was made to define a spectral framework for these objects. Hypernode graphs can also be viewed as an extension of (classical) hypergraphs. The question of learning with hypergraphs has been studied and, for an overview, we refer the reader to [1. In this paper, the authors show that various formulations of the semi-supervised and the unsupervised learning problem on hypergraphs can be reduced to graph problems. For instance, the hypergraph Laplacian of [17] can be defined as a graph Laplacian by an adequate graph construction. To the best of our knowledge, no hypergraph Laplacian which can not be reduced to a graph Laplacian has been defined so far. A very recent tentative to fully use the hypergraph structure was proposed by [7. In this paper, the authors propose to use the hypergraph cut, and they introduce the total variation on a hypergraph as the Lovasz extension of the hypergraph cut. This allows to define a regularization functional on hypergraphs for defining semi-supervised learning algorithms.

\section{Graphs and Hypernode Graphs}

\subsection{Undirected Graphs and Laplacians}

In the following, we recall the commonly accepted definitions of undirected graphs and graph Laplacians. An undirected graph $\mathbf{g}=(V, E)$ is a set of nodes $V$ with $|V|=n$ together with a set of undirected edges $E$ with $|E|=p$. Each edge $e \in E$ is an unordered pair $\{i, j\}$ of nodes and has a non negative weight $w_{i, j}$. In order to define the smoothness of a real-valued node function $f$ over a graph g, we define the gradient function grad for $f$ by, for every edge $(i, j)$,

$$
\operatorname{grad}(f)(i, j)=\sqrt{w_{i, j}}(f(j)-f(i)) .
$$

We can note that $|\operatorname{grad}(f)(i, j)|$ is small whenever $f(i)$ is close to $f(j)$. Then, the smoothness of a real-valued node function $f$ over a graph $\mathbf{g}$ is defined by

$$
\Omega(f)=\sum_{i, j \in V^{2}}|\operatorname{grad}(f)(i, j)|^{2}=f^{T} G^{T} G f,
$$

where $G$ is the matrix of the linear mapping grad from $\mathbb{R}^{n}$ into $\mathbb{R}^{p}$. The symmetric matrix $\Delta=G^{T} G$ is called undirected graph Laplacian, which is also proved to be defined by $\Delta=D-W$ where $D$ is the degree matrix of $\mathbf{g}$ and $W$ the weight matrix of $\mathbf{g} . \Omega(f)=f^{T} \Delta f$ has been used in multiple works (see for example [18, 14]) to ensure the smoothness of a node labeling function $f$.
Additional information concerning the discrete analysis on graphs can be found in [16], which develop a similar theory with a normalized version of the gradient and Laplacian ( $G$ is replaced by $G D^{-1 / 2}$ ).

\subsection{Hypernode Graphs}

The following definition is our contribution to the modeling of binary relationships between sets of entities.

Definition $1 A$ hypernode graph $\mathbf{h}=(V, H)$ is a set of nodes $V$ with $|V|=n$ and a set of hyperedges $H$ with $|H|=p$. Each hyperedge $h \in H$ is an unordered pair $\left\{s_{h}, t_{h}\right\}$ of two non empty and disjoint hypernodes (a hypernode is a subset of $V$ ). Each hyperedge $h \in H$ has a weight function $w_{h}$ mapping every node $i$ in $s_{h} \cup t_{h}$ to a positive weight $w_{h}(i)$ (for $i \notin s_{h} \cup t_{h}$, we define $\left.w_{h}(i)=0\right)$. Each weight function $w_{h}$ of $h=\left\{s_{h}, t_{h}\right\}$ must satisfy the Equilibrium Condition defined by

$$
\sum_{i \in t_{h}} \sqrt{w_{h}(i)}=\sum_{i \in s_{h}} \sqrt{w_{h}(i)} .
$$

An example of hypernode graph is shown in Figure 1 . The red hyperedge links the sets $\{1,4\}$ and $\{2,3\}$. The weights satisfy the Equilibrium condition which ensures that constant node functions have a null gradient as we will see in the next section. The green hyperedge is an unordered pair $\{\{1\},\{3\}\}$ of two singleton sets with weights 0.5 for the nodes 1 and 3 . It can be viewed as an edge between nodes 1 and 3 with edge weight 0.5 . Indeed, when a hyperedge $h$ is an unordered pair $\{\{i\},\{j\}\}$ involving only two nodes, the Equilibrium Condition states that the weights $w_{h}(i)$ and $w_{h}(j)$ are equal. Thus, such a hyperedge can be seen as an edge with edge weight $w_{i, j}=w_{h}(i)=w_{h}(j)$. Therefore, a hypernode graph such that every hyperedge is an unordered pair of singleton nodes can be viewed as an undirected graph, and conversely.

\subsection{Hypernode graph Laplacians}

We will define the smoothness of a real-valued node function $f$ over a hypernode graph with the gradient that we define now.

Definition 2 Let $\mathbf{h}=(V, H)$ be a hypernode graph and $f$ be a real-valued node function, the (hypernode graph) unnormalized gradient of $\mathbf{h}$ is a linear application, denoted by grad, that maps every real-valued node function $f$ into a real-valued hyperedge function 


$$
\begin{aligned}
& \operatorname{grad}(f) \text { defined, for every } h=\left\{s_{h}, t_{h}\right\} \text { in } H, \text { by } \\
& \quad \operatorname{grad}(f)(h)=\sum_{i \in t_{h}} f(i) \sqrt{w_{h}(i)}-\sum_{i \in s_{h}} f(i) \sqrt{w_{h}(i)}
\end{aligned}
$$

where an arbitrary orientation of the hyperedges has been chosen.

As an immediate consequence of the gradient definition and because of the Equilibrium Condition, the gradient of a constant node function is the zero-valued hyperedge function. Also, it can be noted that, for a hyperedge $h \in H,|\operatorname{grad}(f)(h)|^{2}$ is small when the weighted sum of the values $f(i)$ for nodes $i$ in $s_{h}$ is close to the weighted sum of the values $f(j)$ for nodes $j$ in $t_{h}$. Thus, if we denote by $G \in \mathbb{R}^{p \times n}$ the matrix of grad, the smoothness of a real-valued node function $f$ over a hypernode graph $\mathbf{h}$ is defined by $\Omega(f)=f^{T} G^{T} G f$.

Let $\mathbf{h}$ be a hypernode graph with unnormalized gradient $G$, the square $n \times n$ real valued matrix $\Delta=G^{T} G$ is defined to be the unnormalized Laplacian of the hypernode graph $\mathbf{h}$. It should be noted that the Laplacian $\Delta$ does not depend on the arbitrary orientation of the hyperedges used for defining the gradient. When the hypernode graph is a graph, the unnormalized hypernode graph Laplacian matches the unnormalized graph Laplacian. Last, we define the hypernode graph kernel of a hypernode graph $\mathbf{h}$ to be the Moore-Penrose pseudoinverse $\Delta^{\dagger}$ of the hypernode graph Laplacian $\Delta$.

\subsection{Hypernode Graph Laplacians and Learning}

We can characterize hypernode graph Laplacians by

Proposition 1 The class of hypernode graph Laplacians is the class of symmetric positive semidefinite real-valued matrices $M$ such that $\mathbf{1} \in \operatorname{Null}(M)$, where $\operatorname{Null}(M)$ denotes the null space of $M$.

Proof. It is an immediate consequence of the definitions of the hypernode graph gradient and the hypernode graph Laplacian that a hypernode graph Laplacian is a symmetric positive semidefinite real-valued matrix, and that a constant function has a null gradient. For the other direction, let us consider a symmetric positive semidefinite real-valued matrix $M$ such that $\mathbf{1} \in \operatorname{Null}(M)$. Then, consider a square root decomposition $M=G^{T} G$ of $M$. For each line of $G$, one can define a hyperedge $h=\left\{s_{h}, t_{h}\right\}$ with $s_{h}$ the set of nodes with positive values in the line of $G, t_{h}$ the set of nodes with negative values in the line of $G$, and weights equal to the square of values in the line of $G$. The Equilibrium condition is satisfied because $\mathbf{1} \in \operatorname{Null}(M)$ and it is easy to verify that the Laplacian of the resulting hypernode graph $\mathbf{h}$ is $M$.

As a consequence of the construction in the previous proof, it should be noted that there are several hypernode graphs with the same hypernode graph Laplacian because the square root decomposition is not unique. One can also find hypernode graphs whose Laplacian matches that of a graph. One can prove that this is not however the general case. For this, it suffices to consider a hypernode graph Laplacian with an extradiagonal term which is positive. For instance, consider the hypernode graph and its Laplacian matrix $\Delta$ in Figure 2, the Laplacian matrix has 1 as extradiagonal term, thus $\Delta$ is not a graph Laplacian.

As said in Proposition [1 hypernode graph Laplacians are positive semidefinite. This allows to leverage most of the spectral learning algorithms defined in [14, 16, 18, from graphs to hypernode graphs. While it should be noted that hypernode graph Laplacians are stricly more general than graph Laplacians. Consequently, spectral hypernode graph learning can not be reduced to spectral graph learning.

\subsection{Hypernode Graph Laplacians and Signed Graphs}

In this section we present additional properties of hypernode graph kernels. As in the graph case, we have defined the kernel of a hypernode graph to be the Moore-Penrose pseudoinverse of its Laplacian. Because the pseudoinversion preserves semidefiniteness and symmetry, as a consequence of Proposition 1, one can show that the class of hypernode graph kernels is closed under the pseudoinverse operation. As a consequence, the class of hypernode graph kernels is equal to the class of hypernode graph Laplacians. It is worth noticing that the class of graph kernels is not closed by pseudoinversion.

It can also be shown that the class of hypernode graph Laplacians is closed by convex linear combination. This is an important property in the setting of learning from different sources of data. As graph kernels are hypernode graph kernels, it should be noted that the convex linear combination of graph kernels is a hypernode graph kernel, while it is not a graph kernel in general because the class of graph kernels is not closed by convex linear combination. This explains why problems for hypernode graphs can not be solved using graph constructions.

We have shown above that there does not exist in general a graph whose Laplacian is equal to the Laplacian of a given hypernode graph. Nevertheless, given a 


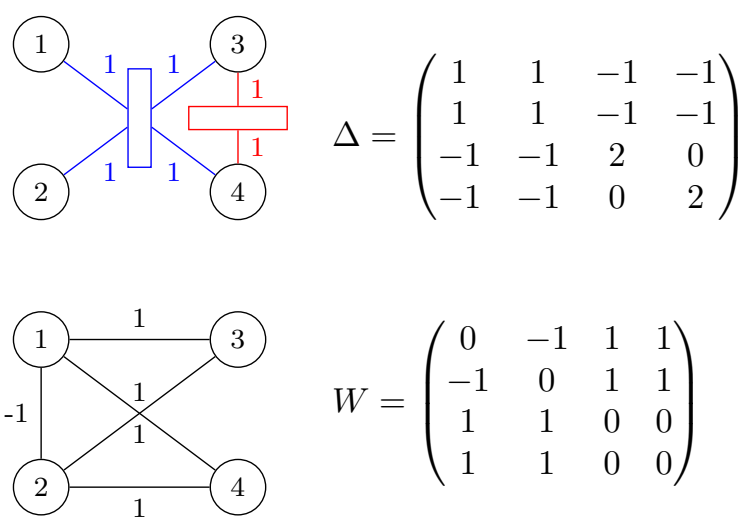

Figure 2 - A hypernode graph, its Laplacian $\Delta$ and its corresponding signed graph (right)

hypernode graph $\mathbf{h}$, one can define a symmetric matrix $W$ of possibly negative weights for pairs of nodes of $\mathbf{h}$ such that the hypernode graph Laplacian of $\mathbf{h}$ is equal to $D-W$, where $D$ is the degree matrix associated with $W$. This means that there is a unique signed graph with weight matrix $W$ such that $D-W$ is the hypernode graph Laplacian of $\mathbf{h}$. The construction is illustrated in Figure 2. This result highlights the subclass of signed graphs whose Laplacian computed with the formula $D-W$ is positive semidefinite. This result also shows that homophilic relations between sets of nodes lead to non homophilic relations between nodes. It can also be shown that the hypernode graph cut defined as the binary restriction of the Laplacian regularization $\Omega(f)=f^{T} \Delta f$ is equivalent to the signed cut defined on the corresponding signed graph. Namely, we have $\forall C \subseteq V, \Omega\left(1_{C}\right)=\sum_{i \in C, j \notin C} W_{i, j}$, where $1_{C}(i)=1$ if and only if $i \in C$ (0 otherwise).

\section{Hypernode Graph Model for Multiple Players Games}

We consider competitive games between two teams where each team is composed of an arbitrary number of players. A first objective is to compute the skill ratings of individual players from game outcomes. A second objective is to predict a game outcome from a batch of games with their outcomes. For that, we will model games by hyperedges assuming that the performance of a team is the sum of the performances of its members as done by the team model proposed in 8 .

\subsection{Multiplayer Games}

Let us consider a set of individual players $P=$ $\{1, \ldots, n\}$ and a set of games $\Gamma=\left\{\gamma_{1}, \ldots, \gamma_{p}\right\}$ between two teams of players. Let us also consider that a player $i$ contributes to a game $\gamma_{j}$ with a non negative weight $w_{j}(i)$. We assume that each player has a skill $s(i)$ and that a game outcome can be predicted by comparing the weighted sum of the skills of the players of each of the two teams. More formally, given two teams of players $A=\left\{a_{1}, a_{2}, \ldots, a_{\ell}\right\}$ and $B=\left\{b_{1}, b_{2}, \ldots, b_{k}\right\}$ playing game $\gamma_{j}$, then $A$ is the winner if and only if

$$
\sum_{i=1}^{\ell} w_{j}\left(a_{i}\right) s\left(a_{i}\right)>\sum_{i=1}^{k} w_{j}\left(b_{i}\right) s\left(b_{i}\right) .
$$

Equivalently, one can rewrite this inequality by introducing a non negative variable $o_{j}$ on the right hand side such that :

$$
\sum_{i=1}^{\ell} w_{j}\left(a_{i}\right) s\left(a_{i}\right)=o_{j}+\sum_{i=1}^{k} w_{j}\left(b_{i}\right) s\left(b_{i}\right),
$$

where $o_{j}$ can be viewed as a variable quantifying the game outcome. In the case of a draw $o_{j}=0$. Given a set of games, it may be impossible to assert that all constraints (1) can be simultaneously satisfied. Our goal is to estimate a skill rating function $s \in \mathbb{R}^{n}$ that respects the game outcomes of the games in $\Gamma$ as much as possible. We define the cost of a game $\gamma_{j}$ with outcome $o_{j}$ for a skill function $s$ by

$$
C_{\gamma_{j}}(s)=\left\|\sum_{i=1}^{\ell} w_{j}\left(a_{i}\right) s\left(a_{i}\right)-\sum_{i=1}^{k} w_{j}\left(b_{i}\right) s\left(b_{i}\right)-o_{j}\right\|^{2} .
$$

Consequently, given a set of games $\Gamma$ and the corresponding game outcomes, the goal is to find a skill rating function $s^{*}$ that minimizes the sum of the different costs, i.e. search for

$$
s^{*}=\arg \min _{s} \sum_{\gamma_{j} \in \Gamma} C_{\gamma_{j}}(s) .
$$

\subsection{Modeling Games with Hypernode Graphs}

In order to model multiplayer games with hypernode graphs, we represent players by nodes, teams by sets of nodes and each game by a hyperedge. Formally, let us consider a game $\gamma_{j}$ between teams $A$ and $B$ in a set of games $\Gamma$. We define the hyperedge $h_{j}$ for game $\gamma_{j}$ as follows 


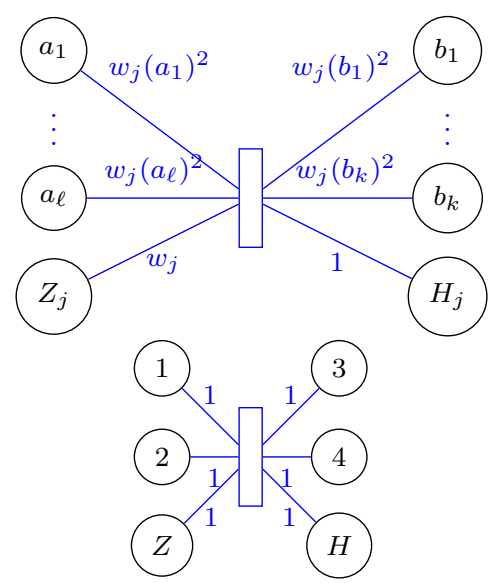

Figure $3-\left[\right.$ top] Generic hyperedge $h_{j}$ for a game $\gamma_{j}$ between team $A=\left\{a_{1}, \ldots, a_{l}\right\}$ and team $B=$ $\left\{b_{1}, \ldots, b_{k}\right\}$ and [bottom] hyperedge $h$ for a game $\gamma$ between team $A=\{1,2\}$ and $B=\{3,4\}$ with node contributions set to 1

1. The players of team A correspond to one of the two hypernodes of $h_{j}$. The weight of a player node is the square of the contribution of the player in the game,

2. do the same construction for team B and the second hypernode of $h_{j}$

3. add a node $H_{j}$, called outcome node to the set of player nodes corresponding to the losing team. The weight of this node is set to 1 ,

4. add a new node $Z_{j}$, called 0 -performance node to the set of player nodes corresponding to the winning team. The weight $w_{j}$ of this node is chosen in order to ensure the Equilibrium condition for the hyperedge $h_{j}$.

A graphical representation of this construction is given in Figure 3 .

Skill rating functions for players are real-valued node functions over the hypernode graph. In order to model the game outcomes in the computation of the skills, we fix some values in the corresponding node function : each 0 -performance node has value 0 ; the outcome node of game $\gamma_{j}$ has the fixed value $o_{j}$ (recall that $o_{j}$ is the outcome of game $\gamma_{j}$, see Eq (2)). As an example, let us consider a game $\gamma$ between two teams $A=\{1,2\}$ and $B=\{3,4\}$ and let us suppose that $A$ wins the game. The corresponding hyperedge $h$ is presented in Figure 3 [bottom]. The skill rating function must satisfy $s(1)+s(2)>s(3)+s(4)$. We consider a node-valued function $f$ on $h$ such that $f(Z)=0$ and $f(H)>0$. The function $f$ is smooth on the hyperedge $h$ if $f(1)+f(2)+f(Z)$ is close to $f(3)+f(4)+f(H)$. Then the smoothness of $f$ allows to express that $f(1)+f(2)>f(3)+f(4)$ and the difference between the two team evaluations relies on the game outcome encoded in $f(H)$. Thus, a smooth realvalued node function on the hyperedge $h$ satisfies the constraints required for a skill rating function for the game $\gamma$.

Hypernode graph for a set of games and the skill rating problem. Let us consider a set of games $\Gamma$, we define the hypernode graph $\mathbf{h}=(V, H)$ as the set of all hyperedges $h_{j}$ for the games $\gamma_{j}$ in $\Gamma$ as defined above. We assume a numbering of $V$ such that $V=$ $\{1, \ldots, N\}$ where $N$ is the total number of nodes, the first $n$ nodes are the player nodes followed by the $t$ 0 -performance nodes and the outcome nodes, that is, $V=\{1, \ldots, n\} \cup\{n+1, \ldots, n+t\} \cup\{n+t+1, \ldots, N\}$. Let $\Delta$ be the unnormalized Laplacian of $\mathbf{h}$, and let $s$ be a real-valued node function on $h . s$ can be seen as a real vector in $\mathbb{R}^{N}$ where the first $n$ entries represent the skills of the $n$ players. The skill rating problem (3) is equivalent to find the optimal vector $s$ solving the optimization problem

$$
\begin{array}{cl}
\underset{s \in \mathbb{R}^{N}}{\operatorname{minimize}} & s^{T} \Delta s \\
\text { subject to } & \forall n+1 \leq j \leq n+t, s(j)=0 \\
& (\text { for } 0 \text {-performance nodes) } \\
& \forall n+t+1 \leq j \leq N, s(j)=o_{j}
\end{array}
$$

(for outcome nodes)

\subsection{Regularizing the hypernode graph}

When the number of games is small, many players will participate to at most one game. Thus, in this case, the number of connected components can be quite large. The player skills in every connected component can be defined independently while satisfying the constraints. Thus, it will be unrelevant to compare player skills in different connected components. In order to solve this issue, we introduce in Equation (4) a regularization term based on the standard deviation of the players skills $\sigma\left(s_{p}\right)$, where $s_{p}=(s(1), \ldots, s(n))$. 
This leads to the new formulation

$$
\begin{array}{cl}
\underset{s \in \mathbb{R}^{N}}{\operatorname{minimize}} & s^{T} \Delta s+\mu \sigma\left(s_{p}\right)^{2} \\
\text { subject to } & \forall n+1 \leq j \leq n+t, s(j)=0 \\
& (\text { for } 0 \text {-performance nodes) } \\
& \forall n+t+1 \leq j \leq N, s(j)=o_{j}
\end{array}
$$

(for outcome nodes),

where $\mu$ is a regularization parameter. Thus, we control the spread of $s_{p}$, avoiding to have extreme values for players participating in a small number of games.

In order to apply graph-based semi-supervised learning algorithms using hypernode graph Laplacians, we now show that the regularized optimization problem can be rewritten as an optimization for some hypernode graph Laplacian. For this, we will show that it suffices to add a regularization node in the hypernode graph $\mathbf{h}$. First, let us recall that if $\bar{s}$ is the mean of the player skills vector $s_{p}=(s(0), \ldots, s(n))$, then for all $q \in \mathbb{R}$ we have

$$
\sigma\left(s_{p}\right)^{2}=\frac{1}{n} \sum_{i=1}^{n}(s(i)-\bar{s})^{2} \leq \frac{1}{n} \sum_{i=1}^{n}(s(i)-q)^{2} .
$$

Thus, in the problem 5, we can instead minimize $s^{T} \Delta s+\frac{\mu}{n} \sum_{i=1}^{n}(s(i)-q)^{2}$ over $s$ and $q$. We now show that this can be written as the minimization of $r^{T} \Delta_{\mu} r$ for some vector $r$ and well chosen hypernode graph Laplacian $\Delta_{\mu}$. For this, let us consider the $p \times N$ gradient matrix $G$ of the hypernode graph $\mathbf{h}$ associated with the set of games $\Gamma$, and let us define the matrix $G_{\mu}$ by

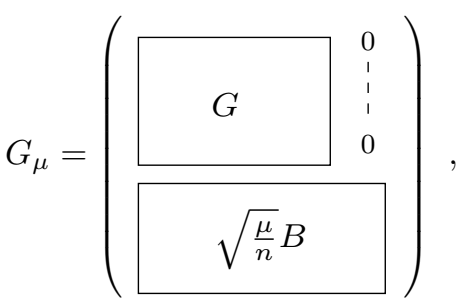

where $B$ is the $n \times(N+1)$ matrix defined by, for every $1 \leq i \leq n, B_{i, i}=-1, B_{i, N+1}=1$, and 0 otherwise. The matrix $G_{\mu}$ is the gradient of the hypernode graph $\mathbf{h}_{\mu}$ obtained from the hypernode graph $\mathbf{h}$ by adding a new node $R$, an hyperedge between every player node and $R$ with node weights $\mu / n$ (such a hyperedge can be viewed as an edge with edge weight $\mu / n)$. The construction is illustrated in Figure 4 with a hypernode graph reduced to a single hyperedge.

Let us denote by $r$ the vector $(s(0), \ldots, s(N), q)$, then since $\Delta=G^{T} G$, we can write

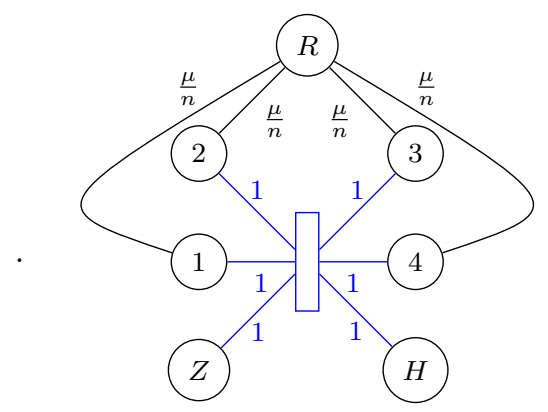

FIGURE 4 - Introducing a regularizer node $R$

$$
r^{T} G_{\mu}^{T} G_{\mu} r=s^{T} \Delta s+\frac{\mu}{n} r B^{T} B r .
$$

As $r B^{T} B r=\sum_{i}\left(s_{i}-q\right)^{2}$, if we denote by $\Delta_{\mu}=$ $G_{\mu}^{T} G_{\mu}$ the $(N+1) \times(N+1)$ unnormalized Laplacian of the hypernode graph $\mathbf{h}_{\mu}$, we can finally rewrite the regularized problem (5) as

$$
\begin{array}{cl}
\underset{r \in \mathbb{R}^{N+1}}{\operatorname{minimize}} & r^{T} \Delta_{\mu} r \\
\text { subject to } & \forall n+1 \leq j \leq n+t, r(j)=0 \\
& (\text { for } 0 \text {-performance nodes) } \\
& \forall n+t+1 \leq j \leq N, r(j)=o_{j}
\end{array}
$$$$
\text { (for outcome nodes) }
$$

\subsection{Infering Skill Ratings and Predic- ting Game Outcomes}

We have shown that predicting skill ratings can be written as the optimization problem (6). It should be noted that it can also be viewed as a semi-supervised learning problem on the hypernode graph $\mathbf{h}_{\mu}$ because the question is to predict node scores (skill ratings) for player nodes when node scores for 0-performance nodes and outcome nodes are given. Using Proposition 1), we get that $\Delta_{\mu}$ is a positive semidefinite real-valued matrix because it is a hypernode graph Laplacian. Therefore, we can use the semi-supervised learning algorithm presented in 18. This algorithm was originally designed for graphs and solves exactly the problem (6) by putting hard constraints on the outcome nodes and on the 0-performance nodes. We denote this method by H-ZGL.

In order to predict skill ratings, another approach is to infer player node scores from 0-performance nodes scores and outcome nodes scores nodes using a regression algorithm. For this, we consider the hypernode graph kernel $\Delta_{\mu}^{\dagger}$ (defined as the Moore-Penrose pseudoinverse of the Laplacian $\Delta_{\mu}$ ) and train a regression 
support vector machine. We denote this method by HSVR.

Using the two previous methods, we can infer skill ratings for players from a given set of games. The inferred skill ratings can be used to predict game outcomes for new games. For this, we suppose that we are given a training set of games $\Gamma_{l}$ with known outcomes together with a set of testing games $\Gamma_{u}$ for which game outcomes are not known. The goal is to predict game outcomes for the testing set $\Gamma_{u}$. Note that other works have considered similar questions in the online setting as in [8, 4] while we consider the batch setting. For the prediction of game outcomes, first we apply a skill rating prediction algorithm presented above given the training set $\Gamma_{l}$ and output a skill rating function $s^{*}$. Then, for each game in $\Gamma_{u}$, we evaluate the inequality (1) with the skills defined by $s^{*}$ and decide the winner. For every player which do not appear in the training set, the skill value is fixed a priori to the mean of known player skills.

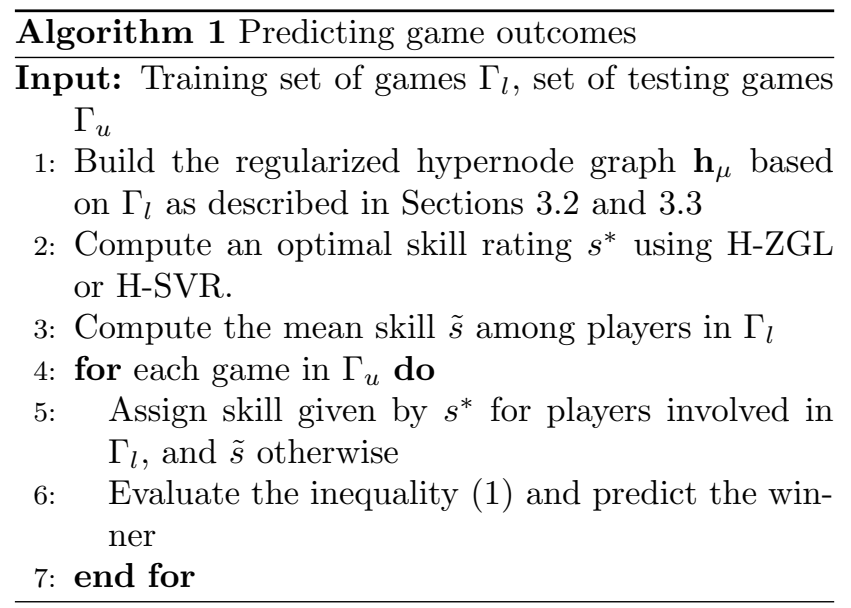

\section{Experiments}

In this section, we report experimental results for the inference of individual skills and the prediction of game outcomes for different datasets.

\subsection{Tennis Doubles}

We consider a dataset of tennis doubles collected between January 2009 and September 2011 from ATP tournaments (World Tour, Challengers and Futures). Tennis doubles are played by two teams of two players. Each game has a winner (no draw is allowed). A game is played in two or three winning sets. The final score corresponds to the number of sets won by each team during the game. The dataset consists in 10028 games with 1834 players.

In every experiment, we select randomly a training subset $\Gamma_{l}$ of games and all remaining games define a testing subset $\Gamma_{u}$. We will consider different sizes for the training set $\Gamma_{l}$ and will compute the outcome prediction error on the corresponding set $\Gamma_{u}$. More precisely, for a given proportion $\rho$ varying from $10 \%$ to $90 \%$, we build a training set $\Gamma_{l}$ using $\rho \%$ of the games chosen randomly among the full game set, the remaining games form the test set $\Gamma_{u}$. We present in Figure 5 and [6] several statistics related to the Tennis dataset. It is worth noticing that many players have played only once. Therefore, the skill rating problem and the game outcome prediction problem become far more difficult to solve when few games are used for learning. Moreover, it should be noted that when the number of games in the training set is small, the number of players in the test set which are involved in a game of the training set is small. In this case many players will have a skill estimated to be the average skill. This explains why the problem is difficult when the number of games in $\Gamma_{l}$ is small.

Given a training set of games $\Gamma_{l}$ and a test set $\Gamma_{u}$, we follow the experimental process described in Algorithm 1. For the definition of the hypergraph, we fix all player contributions in games to 1 because we do not have additional information than final scores. Thus the player nodes weights in every hyperedge is 1 . In the optimization problem 6, the game outcomes $o_{j}$ are defined to be the difference between the number of sets won by the two teams. This allows to take account of the score when computing player skills. In order to reduce the number of nodes in our hypernode graph, we merge all the 0-performance nodes in a single one that is shared by all the hyperedges. We do the same for outcome nodes because score differences can be 1,2 or 3 . The resulting hypernode graph has at most 1839 nodes : at most 1834 player nodes, 1 shared 0-performance node, 3 outcome nodes, and 1 regularizer node.

To complete the definition of the hypernode graph $\mathbf{h}_{\mu}$ constructed from the game set $\Gamma_{l}$, it remains to fix the parameter value for $\mu / n$. For this, assuming a Gaussian distribution for skill ratings and comparing expected values for the two terms $s^{T} \Delta s$ and $\mu \sigma\left(s_{p}\right)^{2}$, we show (see Appendix A) that the value of $\mu / n$ should have the same order of magnitude than the average number of games played by a player. We fix the default value to be 16 for $\mu / n$ and use this default value in all experiments.

Given $\mathbf{h}_{\mu}$, following Algorithm 1, we apply the skill 


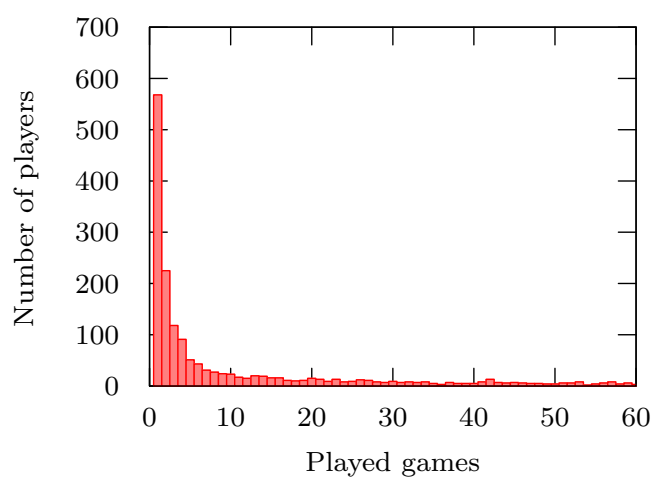

Figure 5 - Distribution of the number of players against the number of played games

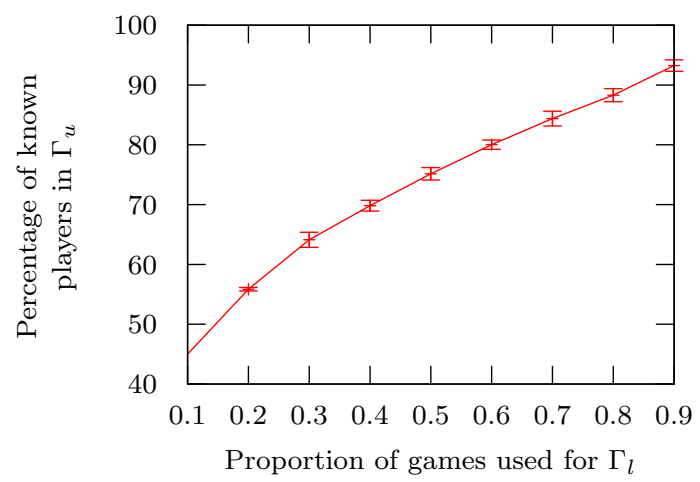

FiguRE 6 - Average percentage of players in $\Gamma_{u}$ which are involved in some game in $\Gamma_{l}$

rating prediction algorithms H-ZGL and H-SVR. In order to compare our method, we also infer skill ratings using Elo Duelling and Trueskill $[8]^{1}$ Then, we predict game outcomes from the inferred skill ratings. The results are given in Figure 7 (for each value of $\rho$, we repeat the experiment 10 times). It can be noted that Elo duelling performs poorly. Also, it can be noted that H-ZGL is significantly better than Trueskill whatever is the chosen proportion.

\subsection{Xbox Title Halo2}

The Halo2 dataset was generated by Bungie Studio during the beta testing of the XBox title Halo2. It has been notably used in [8] to evaluate the performance of the Trueskill algorithm. We consider the Small Teams

1. TrueSkill and Elo implementations are from [6]. Results were double-checked using 13 and 12 . Parameters of Elo and TrueSkill are the default parameters of [6] $(K=32$ for Elo, $\mu_{0}=25, \beta=12.5, \sigma=8.33$ and $\tau=0.25$ for TrueSkill).

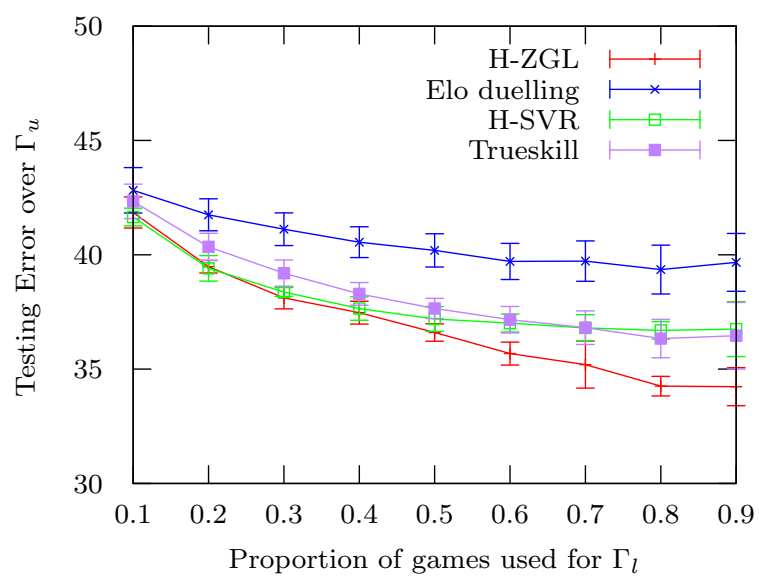

Figure 7 - Predictive error depending on the proportion of games used to build $\Gamma_{l}$

dataset with 4992 players and 27536 games opposing up to 12 players in two teams. It should be noted that a game can confront two teams of different size. Each game can result in a draw or a win of one of the two teams. The proportion of draws is $22.8 \%$. As reported in $[8$, the prediction of draws is challenging and it should be noted that Trueskill and our algorithm fail to outperform a random guess for the prediction of draw.

We again consider the experimental process described in Algorithm 1. As for the Tennis dataset, we fix all players contributions in games to 1 . In the optimization problem 6, the game outcomes $o_{j}$ are defined to be equal to 1 when the game has a winner and 0 otherwise. This is because game scores in the dataset vary depending on the type of game considered. As above, we merge the 0 -performance nodes into a single one and do the same for outcome nodes. The value of $\mu / n$ is again set to 16 .

We use the experimental process defined for the Tennis dataset in order to compare the skill rating algorithms H-ZGL, H-SVR, Elo Duelling and Trueskill. The number of prediction errors for game outcomes is computed assuming that a draw can be regarded as half a win, half a loss [11. We present the experimental results in Figure 8. For a proportion of $10 \%$ of games in the training set, H-ZGL, H-SVR and Trueskill give similar results. While with larger training sets, our hypernode graph learning algorithms outperform Trueskill. On the Small Teams dataset, the inference of the skill rating function with hypernode graphs is achieved in less than 2 minutes. 


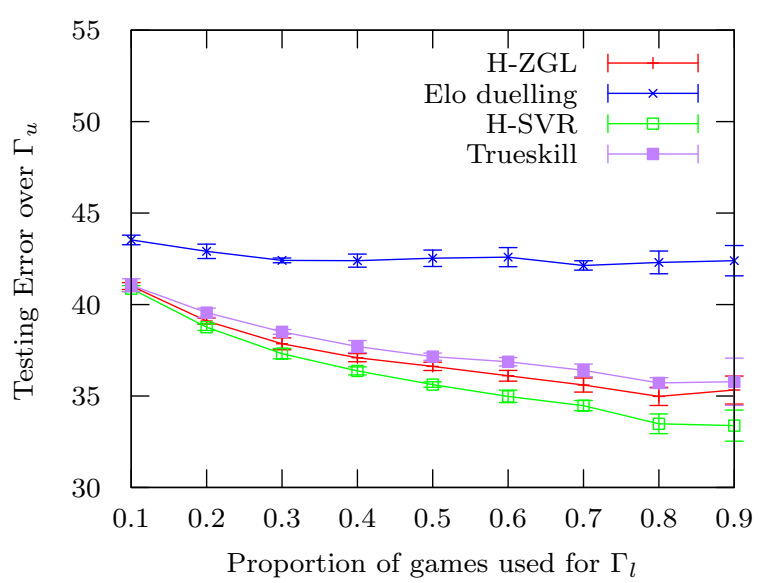

FiguRE 8 - Predictive error depending on the proportion of games used to build $\Gamma_{l}$

\section{Conclusion}

We have introduced a new class of undirected hypergraphs modeling pairwise relationships between sets of individuals. The class of hypernode graphs strictly extends the class of graphs while keeping important properties for the design of machine learning algorithms. For instance, Laplacians and kernels have been generalized to hypernode graphs allowing to define semisupervised learning algorithms for hypernode graphs in the spirit of [16. We have applied our work to the problem of skill rating and game outcome prediction in multiple players games and obtained very promising results.

From a theoretical perspective, it should be interesting to study more deeply the notion of cut for our class of hypernode graphs. Recent work by [7] could be a promising line of research in order to design an adequate definition of cuts. From a machine learning perspective, we should define online learning algorithms for hypernode graphs following 9]. Also, we should consider normalized Laplacians. Last, we are confident in the capability of our model to handle new applications in networked data.

\section{Références}

[1] Sameer Agarwal, Kristin Branson, and Serge Belongie. Higher order learning with graphs. In Proc. of ICML, pages 17-24, 2006.

[2] Claude Berge. Graphs and hypergraphs. NorthHoll Math. Libr. North-Holland, Amsterdam,
1973.

[3] Jie Cai and Michael Strube. End-to-end coreference resolution via hypergraph partitioning. In Proc. of COLING, pages 143-151, 2010.

[4] Arpad Emrick Elo. The Rating of Chess Players, Past and Present. Arco Publishing, 1978.

[5] Giorgio Gallo, Giustino Longo, Stefano Pallottino, and Sang Nguyen. Directed hypergraphs and applications. Discrete Applied Mathematics, 42(23) :177-201, 1993.

[6] Scott Hamilton. PythonSkills : Implementation of the trueskill, glicko and elo ranking algorithms. https://pypi.python.org/pypi/skills, 2012.

[7] Matthias Hein, Simon Setzer, Leonardo Jost, and Syama Sundar Rangapuram. The total variation on hypergraphs - learning on hypergraphs revisited. In Proc. of NIPS, pages 2427-2435, 2013.

[8] Ralf Herbrich, Tom Minka, and Thore Graepel. Trueskill $^{\text {tm }}$ : A bayesian skill rating system. In Proc. of NIPS, pages 569-576, 2006.

[9] Mark Herbster and Massimiliano Pontil. Prediction on a graph with a perceptron. In Proc. of NIPS, pages 577-584, 2006.

[10] Steffen Klamt, Utz-Uwe Haus, and Fabian Theis. Hypergraphs and cellular networks. PLoS Computational Biology, 5(5), May 2009.

[11] Jan Lasek, Zoltán Szlávik, and Sandjai Bhulai. The predictive power of ranking systems in association football. International Journal of Applied Pattern Recognition, 1(1) :27-46, 2013.

[12] Heungsub Lee. Python implementation of Elo : A rating system for chess tournaments. https: //pypi.python.org/pypi/elo/0.1.dev, 2013.

[13] Heungsub Lee. Python implementation of TrueSkill : The video game rating system. http:// trueskill.org/, 2013.

[14] Ulrike Von Luxburg. A tutorial on spectral clustering. Statistics and computing, 17(4) :395-416, 2007.

[15] Shujun Zhang, Geoffrey D. Sullivan, and Keith D. Baker. The automatic construction of a viewindependent relational model for 3 -d object recognition. Pattern Analysis and Machine Intelligence, IEEE Transactions on, 15(6) :531-544, 1993.

[16] Dengyong Zhou, Jiayuan Huang, and Bernhard Schölkopf. Learning from labeled and unlabeled data on a directed graph. In Proc. of ICML, pages 1036-1043, 2005. 
[17] Dengyong Zhou, Jiayuan Huang, and Bernhard Schölkopf. Learning with hypergraphs : Clustering, classification, and embedding. In Proc. of NIPS, pages 1601-1608, 2007.

[18] Xiaojin Zhu, Zoubin Ghahramani, John Lafferty, et al. Semi-supervised learning using gaussian fields and harmonic functions. In Proc. of ICML, volume 3, pages 912-919, 2003. 